\title{
Statics and Dynamics of Phase Segregation in Multicomponent Fermion Gas
}

\author{
K. Esfarjanit, S.T. Chuit, V. Kumar*⿻⺀, and Y. Kawazoe \\ Institute for Materials Research, Tohoku University, Sendai 980-8577, Japan
}

\begin{abstract}
We investigate the statics and dynamics of spatial phase segregation process of a mixture of fermion atoms in a harmonic trap using the density functional theory. The kinetic energy of the fermion gas is written in terms of the density and its gradients. Several cases have been studied by neglecting the gradient terms (the Thomas-Fermi limit) which are then compared with the MonteCarlo results using the full gradient corrected kinetic energy. A linear instability analysis has been performed using the random-phase approximation. Near the onset of instability, the fastest unstable mode for spinodal decomposition is found to occur at $q=0$. However, in the strong coupling limit, many more modes with $q \approx K_{F}$ decay with comparable time scales.
\end{abstract}

\section{INTRODUCTION}

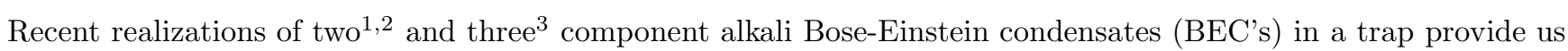
with new systems to explore the physics in otherwise unachievable parameter regimes. recently been observed in the phase segregation dynamics of mixtures of $\mathrm{Rb}$. 2 and $\mathrm{Na} 3$ gases. Periodic spatial structures were found at intermediate times which then recombine at a later time.

Phase segregation phenomena have been much studied in materials science and these can be understood using classical mechanics. Spatial modulations have also been observed, for example, in AlNiCo alloys 7 These were explained in terms of a concept called spinodal decomposition. 6 When a system is quenched from the homogeneous phase into a broken-symmetry phase, the ordered phase does not order instantaneously. Instead, different length scales set in as the domains form and grow with time. For the BEC's, however, quantum mechanics play an important role. It has been shown $\mathrm{g}$ that it is possible to have an analogous spinodal decomposition, which manifests some of the phenomenology including a periodic spatial structure at an intermediate time that is now determined by quantum mechanics The time scale provides for a self-consistent check of the theory and is consistent with the experimental results. 3 The growth of domains at later times is now determined by quantum tunneling and not by classical diffusion.

Recently, it became possible 10 to cool a single component system of about a million ${ }^{40} \mathrm{~K}$ fermionic atoms in a magnetic trap below the Fermi temperature, $T_{F}$, leading to the realization of a spin-polarized fermion gas of atoms. Similar to electrons in a solid, the dilute gas of atoms fills all the lowest energy states below the Fermi energy, $E_{F}$. The transition to this quantum degenerate state is gradual as compared to the abrupt phase transition into a Bose condensate. For single component fermionic systems, however, the equilibrium is difficult to achieve as the $s$-wave elastic collisions are prohibited due to Pauli exclusion principle. In the experiments of DeMarco and Jin $\mathbb{L}^{0}$, this was circumvented by using a mixture of two nuclear spin states of ${ }^{40} \mathrm{~K}$ atoms for which $s$-wave collisions are allowed. One of the manifestations of quantum mechanics was the nature of momentum distribution which differed from the well known classical gaussian distribution. This system corresponds to the weak coupling limit in which the physical properties are close to those of a non-interacting fermion gas. The other system which is being explored ${ }^{11}$ is the gas of ${ }^{6} \mathrm{Li}$ atoms. Mixtures of fermions interating with the Coulomb interaction have been studied in the context of the electron-hole fluid12. For fermions mixtures on a lattice site interacting with the Hubbard Hamiltonian, the partial phase segregation leads to antiferromagnetism. Thermodynamic properties as well as density andmomentum distributions of spin-polarized fermionic gas of atoms in a harmonic trap have been studied in recent years 13.41 .5 . Butts and Rokhsar 13 have obtained universal forms of the spatial and momentum distributions for a single component spinpolarized non-interacting fermion gas using the Thomas-Fermi (TF) approximation, whereas Schneider and Wallis 15 have studied the effects of shell closure for small number of atoms, similar to the nuclear shell model. Bruun and Burnett 14 have studied an interacting fermion gas of ${ }^{6} \mathrm{Li}$ atoms which have a large negative scattering length. Such an interaction could also lead to the possibility of superfluid state 16 in these systems. In the present paper, we consider mixtures of these new finite systems of ultracold fermionic atoms with a positive scattering length in the limit of both weak and strong coupling and explore the equilibrium and non-equilibrium quantum statistical physics using the TF approximation, Monte Carlo simulations, and the random phase approximation.

In section II we present the equilibrium static properties of mixtures of fermionic atoms in different parameters regimes using both the TF and the Monte Carlo simulations. In section III, we study the dynamics of phase segregation of such mixtures using a linear stability analysis. Finally, conclusions will be presented in section IV. 


\section{STATICS}

We first start with the statics of a two component fermion gas of atoms with masses $m_{1}$ and $m_{2}$ and particle numbers $N_{1}$ and $N_{2}$. This is assumed to be confined in an azimuthally symmetric harmonic trap with radial and axial frequencies $\omega$ and $\lambda \omega$, respectively which are considered to be the same for both the components. Unlike the electron gas in matter, the fermion gas of atoms is neutral and dilute. Therefore, significant interactions between atoms are only short-ranged and that would be responsible for any phase segregation in the system. In the long wavelength limit, the system can be well described by the density functional theory and the total energy can be written as

$$
E=\int\left[\sum_{\sigma} E_{0 \sigma}\left(\rho_{\sigma}\right)+g \rho_{1}(\mathbf{r}) \rho_{2}(\mathbf{r})\right] \mathrm{d} \mathbf{r} .
$$

Here $E_{0 \sigma}=\frac{\hbar^{2}}{2 m_{\sigma}} \tau_{\sigma}(\mathbf{r})+\frac{1}{2} m_{\sigma} \omega^{2}\left(x^{2}+y^{2}+\lambda^{2} z^{2}\right) \rho_{\sigma}(\mathbf{r})$ is the non-interacting part of the energy density and $\rho_{\sigma}(\mathbf{r})$ is the particle density of the component $\sigma=1,2$ with $\int \rho_{\sigma}(\mathbf{r}) d \mathbf{r}=N_{\sigma}$. The interaction term has been approximated by the contact potential $g \delta\left(\mathbf{r}-\mathbf{r}^{\prime}\right)$. $g$ is related to the scattering length $a$ by $g=2 \pi \hbar^{2} a / \bar{m}$, with $\bar{m}=m_{1} m_{2} /\left(m_{1}+m_{2}\right)$. In accordance with the experiments, we take $a$ to be positive and consider only the $s$-wave scattering. Therefore, the contribution to the interaction term is non-zero only when the species are different or are in different hyperfine states as in experiments. From the Pauli exclusion principle, there is no contact interaction between particles of the same species (spin). In a more general treatment including $p$-wave scattering there would be additional terms involving interaction between identical species also. But these are small, and thus neglected.

For the kinetic energy density $\tau_{\sigma}$ we use a local approximation including the first and second derivatives of the particle density,

$$
\tau_{\sigma}(\mathbf{r})=\frac{3}{5}\left(6 \pi^{2}\right)^{2 / 3} \rho_{\sigma}(\mathbf{r})^{5 / 3}+\frac{1}{36} \frac{\left|\nabla \rho_{\sigma}(\mathbf{r})\right|^{2}}{\rho_{\sigma}(\mathbf{r})}+\frac{1}{3} \nabla^{2} \rho_{\sigma}(\mathbf{r})
$$

The first term represents the Thomas-Fermi (TF) approximation to the kinetic energy. The second term is $\frac{1}{9}\left|\nabla \sqrt{\rho_{\sigma}}\right|^{2}$ and represents the gradient correction to the kinetic energy. The integral of the third term extended to infinity vanishes, and thus it will not be included in the calculations. The Monte-Carlo results confirm that the gradient term is at least 2 orders of magnitude smaller than the TF term, but this term is important in that it can break the symmetry of the ground state and lead to asymmetric states with a lower energy .

Without the interaction term in (1), the system behaves in the same fashion as the one component system for which Butts and Rokhsar 13 obtained $E_{F}$ to be related to the total particle number $N$ by $E_{F}=\hbar \omega(6 \lambda N)^{1 / 3}$. Defining $R_{F}=\left(2 E_{F} / m \omega^{2}\right)^{1 / 2}$ (giving the characteristic size of the gas), and $K_{F}=\left(2 m E_{F} / \hbar^{2}\right)^{1 / 2}$ (momentum of a free particle of energy $E_{F}$ ), they calculated the density profile at $\mathrm{T}=0$ to be given by

$$
\rho_{\text {non-interacting }}(\mathbf{r})=\rho_{0}\left[1-\bar{r}^{2} / R_{F}^{2}\right]^{3 / 2},
$$

with $\bar{r}^{2}=x^{2}+y^{2}+\lambda^{2} z^{2}, \rho_{0}=8 N \lambda / \pi^{2} R_{F}^{3}=K_{F}^{3} / 6 \pi^{2}$. In the TF approximation, the trapping potential can be treated to be locally constant and we can define a local Fermi wavevector, $k_{F}(\mathbf{r})$ so that $E_{F}=\hbar^{2} k_{F}^{2}(\mathbf{r}) / 2 m+V(\mathbf{r})$, and the density at $\mathrm{T}=0$ can also be written as $\rho_{\text {non-interacting }}(\mathbf{r})=k_{F}^{3}(\mathbf{r}) / 6 \pi^{2}$.

We now examine the properties of the mixed (two-component) interacting system and will show how the repulsive interaction modifies this non-interacting density profile as well as other properties of the system. The strength of the coupling, which controls the phase segregation, depends on the dimensionless parameter which is the ratio between the interaction and the kinetic energies, namely $g \rho_{1} \rho_{2} /\left[\frac{3 \hbar^{2}}{10}\left(6 \pi^{2}\right)^{2 / 3}\left(\rho_{1}^{5 / 3} / m_{1}+\rho_{2}^{5 / 3} / m_{2}\right)\right]$. In the simple case of equal masses $\left(m_{1}=m_{2}=m\right)$ and densities $\left(\rho_{1}=\rho_{2}=\rho\right)$ of the two components, this simply scales as $a K_{F}$. This means that the coupling would be stronger if $a$ or the density is large. Also as $E_{F}$ is proportional to the frequency of the trap at constant $N$ (a higher frequency leads to a larger separation between the levels), the coupling would be large for higher frequencies. From now on, to measure the strength of the interaction, we will use the dimensionless parameters $c_{\sigma}=K_{F \sigma} a / \pi$, where $K_{F \sigma}=\left(2 m_{\sigma} \mu_{\sigma}\right)^{1 / 2} / \hbar$, or in the case of equal chemical potentials, just $c=K_{F} a / \pi$.

For a general two-component system with chemical potentials $\mu_{1}$ and $\mu_{2}$, the ground state is obtained by minimizing the thermodynamic potential $\Omega=E-\int\left(\mu_{1} \rho_{1}+\mu_{2} \rho_{2}\right) \mathrm{d} \mathbf{r}$. This leads to the following system of equations:

$$
\begin{aligned}
& \frac{\partial \Omega}{\partial \rho_{1}(\mathbf{r})}=\frac{\hbar^{2}}{2 m_{1}}\left[\left(6 \pi^{2} \rho_{1}\right)^{\frac{2}{3}}-\frac{1}{36}\left(\left|\frac{\nabla \rho_{1}}{\rho_{1}}\right|^{2}+2 \frac{\nabla^{2} \rho_{1}}{\rho_{1}}\right)\right]+\left(\frac{1}{2} m_{1} \omega^{2} \bar{r}^{2}-\mu_{1}+g \rho_{2}\right)=0 \\
& \frac{\partial \Omega}{\partial \rho_{2}(\mathbf{r})}=\frac{\hbar^{2}}{2 m_{2}}\left[\left(6 \pi^{2} \rho_{2}\right)^{\frac{2}{3}}-\frac{1}{36}\left(\left|\frac{\nabla \rho_{2}}{\rho_{2}}\right|^{2}+2 \frac{\nabla^{2} \rho_{2}}{\rho_{2}}\right)\right]+\left(\frac{1}{2} m_{2} \omega^{2} \bar{r}^{2}-\mu_{2}+g \rho_{1}\right)=0 .
\end{aligned}
$$


Similar to the one-component case, one can rewrite the above in a dimensionless form by introducing for each of the species $\sigma$, the following quantities: $R_{\sigma}=\left[2 \mu_{\sigma} / m_{\sigma} \omega^{2}\right]^{\frac{1}{2}}, \rho_{\sigma 0}=K_{F \sigma}^{3} / 6 \pi^{2}, \mathcal{G}_{\sigma}=g \rho_{\bar{\sigma} 0} / \mu_{\sigma}$, and $n_{\sigma}(\mathbf{r})=\rho_{\sigma}(\mathbf{r}) / \rho_{\sigma 0}$. Here $\bar{\sigma}=3-\sigma$. If one neglects the smaller terms containing derivatives of $\rho$ (the TF limit), one obtains the following algebraic equations satisfied by the dimensionless densities $n_{1}$ and $n_{2}$ for any coupling strength $\mathcal{G}_{\sigma}$ :

$$
\begin{aligned}
& n_{1}^{2 / 3}=1-\bar{r}^{2} / R_{1}^{2}-\mathcal{G}_{1} n_{2} \\
& n_{2}^{2 / 3}=1-\bar{r}^{2} / R_{2}^{2}-\mathcal{G}_{2} n_{1} .
\end{aligned}
$$

We see that the effect of the additional $\mathcal{G}_{\sigma} n_{\bar{\sigma}}$ term, i.e. the interaction, is to deplete the regions where $n_{\bar{\sigma}}$ is highest (without necessarily leading to a phase segregation).

When there is phase segregation, the interface energy is proportional to the square root of the coefficient of the gradient term 17 and it often serves to distinguish different configurations. In that case, their effect cannot be neglected and these are included in the Monte Carlo simulations. We next discuss some special cases in the TF limit.

\section{A. TF limit: Similar densities: $\left(\mu_{1}=\mu_{2}\right)$ for any coupling}

To simplify the notations, we will use: $\mu_{1}=\mu_{2}=\mu ; R_{1}=R_{2}=R ; \mathcal{G}_{1}=\mathcal{G}_{2}=\mathcal{G}$. In this case, three solutions to Eq. (6) will correspond to $n_{1}=n_{2}$, of which only one is physical with $n_{1}>0$. If a solution $n_{2}=f\left(n_{1}\right)$ exists, by symmetry, the other one is necessarily $n_{1}=f\left(n_{2}\right)$. These solutions with $n_{1} \neq n_{2}$ can be obtained numerically. The real solutions are plotted in Fig. 1, where the $n_{1}=n_{2}$ solution is referred to as "Sym", and the other conjugate (asymmetric) solutions are referred to as "A1" and "A2". Below we discuss these solutions in the weak and strong coupling limits.

\section{Weak or intermediate coupling regime}

In this case we look for symmetric solutions $\left(n_{1}=n_{2}=n\right)$. Equation $(6)$ then reduces to (dropping the subscripts):

$$
n(\mathbf{r})^{2 / 3}=1-\bar{r}^{2} / R^{2}-\mathcal{G} n(\mathbf{r})
$$

which can be solved easily numerically to give the density profile of the non-segregated phase. It is possible to show that after proper rescaling, the result for all coupling strengths and at any point can be summarized in a single universal curve in Fig. 1. If $n(\mathbf{r})$ is a solution to Eq. (7), then $\mathcal{N}=n \mathcal{G}^{3}$ versus $\mathcal{P}=\left[1-\bar{r}^{2} / R^{2}\right] \mathcal{G}^{2}$ is the universal function of Fig. 1 satisfying $\mathcal{N}^{2 / 3}+\mathcal{N}-\mathcal{P}=0$. For small couplings and near the boundary $\left(\mathcal{P} \approx 0 ; \mathcal{N}^{2 / 3} \gg \mathcal{N} \Leftrightarrow \mathcal{N}=\mathcal{P}^{3 / 2}\right.$ ), this curve is a power law and in fact tends to the non-interacting density $n(\mathbf{r}) \approx\left[1-\left(x^{2}+y^{2}+\lambda^{2} z^{2}\right) / R^{2}\right]^{3 / 2}$.

\section{Strong coupling regime}

The above situation, however, can not be always sustained. In the strong coupling limit, we can have phase segregation $\left(n_{1} \neq n_{2}\right)$, and one needs to go back to Eq. (6) which now admits lower energy solutions that are not "permutation symmetric":

$$
\begin{aligned}
& n_{1}^{2 / 3}+\mathcal{G} n_{2}=1-\left(x^{2}+y^{2}+\lambda^{2} z^{2}\right) / R^{2} \Leftrightarrow \mathcal{N}_{1}^{2 / 3}+\mathcal{N}_{2}=\mathcal{P} \Leftrightarrow \mathcal{N}_{1}^{2}=\left(\mathcal{P}-\mathcal{N}_{2}\right)^{3} \\
& n_{2}^{2 / 3}+\mathcal{G} n_{1}=1-\left(x^{2}+y^{2}+\lambda^{2} z^{2}\right) / R^{2} \Leftrightarrow \mathcal{N}_{2}^{2 / 3}+\mathcal{N}_{1}=\mathcal{P} \Leftrightarrow \mathcal{N}_{2}^{2}=\left(\mathcal{P}-\mathcal{N}_{1}\right)^{3}
\end{aligned}
$$

where we used the same simplifying notations as before. As previously mentioned, the symmetric solution $\mathcal{N}_{1}=\mathcal{N}_{2}$ always exists. This can be exploited to reduce the above equations to a quadratic equation, which is analytically more transparent.

Subtracting the above equations from each other and dividing out by $\mathcal{N}_{1}-\mathcal{N}_{2}$, we obtain,

$$
\mathcal{N}_{1}+\mathcal{N}_{2}=\left(\mathcal{P}-\mathcal{N}_{2}\right)^{2}+\left(\mathcal{P}-\mathcal{N}_{1}\right)^{2}+\left(\mathcal{P}-\mathcal{N}_{2}\right)\left(\mathcal{P}-\mathcal{N}_{1}\right)
$$

This quadratic equation can be solved for $\mathcal{N}_{1}$ in terms of $\mathcal{N}_{2}$.

The solutions will all be axially symmetric in that they are functions of $\bar{r}^{2}$ only. In actuality, the axial symmetry can also be broken, but we do not find it here since we neglected the terms in gradient of the particle density in 
the kinetic energy. The broken symmetry solutions will be discussed in the subsection $\mathrm{E}$ where we present results obtained from the Monte Carlo simulations incorporating these terms. In Fig. 1, the solutions with $n_{1} \neq n_{2}$ can be seen in the limit of small reduced distance and large $\mathcal{P}$. The bifurcation point where these solutions start to occur, corresponds, from numerical results, to $\mathcal{P}_{c} \approx 0.741$, and $\mathcal{N}_{c}=n \mathcal{G}^{3} \approx 0.296$, which separate the strong coupling regime from the weak one. In both figures, the symmetric solution is drawn with solid line, and the asymmetric ones with dashed lines. Actually, at the bifurcation point, we have exactly $\mathcal{G} n^{\frac{1}{3}}=2 / 3$ as will be shown in the TF linear stability analysis section below. Since $\mathcal{G}^{2}=\mathcal{P} /\left(1-\bar{r}^{2} / R^{2}\right) \geq \mathcal{P}$, the smallest coupling $\mathcal{G}_{c}$ for the unequal solutions to occur satisfies $\mathcal{G}_{c}=\sqrt{\mathcal{P}_{c}}$. Since $\mathcal{G}=(4 / 3) K_{F} a / \pi$, we find a critical dimensionless coupling $c=\left(K_{F} a / \pi\right)_{c} \approx 0.646$. We shall come back and compare this value with that obtained with a different approach.

\section{B. TF limit: Very different densities: $\left(\mu_{1} \gg \mu_{2}\right)$ for any coupling}

One can also treat the case where one of the species is a minority $\left(\mu_{1} \gg \mu_{2}\right)$. If we assume $\mu_{1}=\lambda^{2} \mu_{2}$, then $R_{1}=\lambda R_{2} ; K_{F 1}=\lambda K_{F 2} ; \rho_{10}=\lambda^{3} \rho_{20} ; \mathcal{G}_{2}=\lambda^{5} \mathcal{G}_{1}$, and $n_{\sigma} \sim 1$. The density distribution of the majority species will be weakly perturbed. Referring to Eqs. (6), one can see that the coupling $\mathcal{G}_{1}=g \rho_{20} / \mu_{1}$ becomes very small and

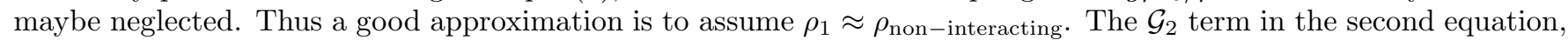
however, is a large quantity, and will strongly affect the particle density $n_{2}$. Therefore,

$$
n_{2}(\mathbf{r}) \approx\left[1-\bar{r}^{2} / R_{2}^{2}-\mathcal{G}_{2}\left[1-\bar{r}^{2} / R_{1}^{2}\right]^{\frac{3}{2}}\right]^{\frac{3}{2}}
$$

In the presence of the majority species, the number of atoms of minority species will be much less than their noninteracting counterparts with the same chemical potential. As we can see from the above equation, their number, even at the origin is reduced by a factor of $\left(1-\mathcal{G}_{2}\right)^{\frac{3}{2}}$. We find that for a large enough $\mathcal{G}_{2}$ the density $\mathcal{N}_{2}$ is depleted

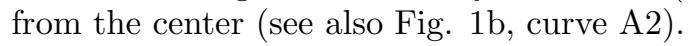

\section{TF limit: linear instability analysis}

We next study the fluctuations of the system about its equilibrium configuration in the TF limit by expanding the thermodynamic potential $\Omega$ upto second order in the particle density variation $\delta \rho$ about its minimum which was computed above. The sign of the second derivative of $\Omega$ will decide the stability of the symmetric phase. A phase segregation occurs when the Hessian (second derivative matrix) ceases to be positive definite. If the transition is first order, it would have already occurred before reaching a negative second derivative. The second derivative from Eqs. (3) and (4) is just a $2 \times 2$ matrix:

$$
\frac{\partial^{2} \Omega}{\partial \rho_{\sigma} \partial \rho_{\sigma^{\prime}}}=\frac{\hbar^{2}}{2 m_{\sigma}} \frac{2}{3}\left(6 \pi^{2}\right)^{\frac{2}{3}} \rho_{\sigma}^{-\frac{1}{3}} \delta_{\sigma \sigma^{\prime}}+g\left(1-\delta_{\sigma \sigma^{\prime}}\right)
$$

The phase instability criterion thus becomes $\omega_{-}=0$ where $\omega_{-}$is the smallest eigenvalue of the Hessian matrix; implying:

$$
\frac{\hbar^{2}}{2 \sqrt{m_{1} m_{2}}} \frac{2}{3}\left(6 \pi^{2}\right)^{\frac{2}{3}}\left(\rho_{1} \rho_{2}\right)^{-\frac{1}{6}}=g \Leftrightarrow \frac{\mu}{\rho_{0}} \frac{2}{3} n^{-1 / 3}=g \text { if }\left(\rho_{1}=\rho_{2}\right)
$$

Thus, in the symmetric case $\left(\mu_{1}=\mu_{2} ; \rho_{1}=\rho_{2}\right)$, the instability will first occur locally at the point where the relation $\mathcal{N}^{1 / 3}=\mathcal{G} n^{\frac{1}{3}}=2 / 3$ is satisfied. This implies that $\mathcal{N}=0.296$, which is exactly the critical $\mathcal{N}_{c}$ obtained earlier from a different analysis. These two instabilities occuring at the same point suggest that, within the adopted model (TF), the transition might be of second order.

\section{Possibility of density modulation instability}

Similar to the electron gas which has several kinds of instabilities such as ferromagnetism, antiferromagnetism, charge density wave, superconductivity, etc... these two-component systems might also exhibit other types of instabilities. To investigate them, we will assume the homogeneous case $(\omega=0)$ as the analysis can be made simpler by 
using the Fourier decomposition of the density. To get some understanding of the nonuniform systems (such as in a trap), one can assume in a semiclassical approximation, that the Fermi momentum depends on the position, as before.

The density for the species $\sigma$ can be written as the sum of its Fourier components: $\rho_{\sigma}(\mathbf{r})=\bar{\rho}_{\sigma}+\sum_{\mathbf{q} \neq 0} \rho_{\sigma \mathbf{q}} e^{i \mathbf{q} . \mathbf{r}}$, with $\bar{\rho}_{\sigma} \gg \rho_{\sigma \mathbf{q}}$. Substituting this expression in the thermodynamic potential $\Omega$, expanding up to second powers of $\rho_{\sigma \mathbf{q}}$, and minimizing $\Omega$ with respect to the Fourier components, we obtain:

$$
\begin{gathered}
\frac{\partial \Omega}{\partial \rho_{\sigma \mathbf{q}}}=\frac{\hbar^{2}}{2 m_{\sigma}}\left[\frac{2}{3}\left(6 \pi^{2} \bar{\rho}_{\sigma}\right)^{\frac{2}{3}} \frac{\rho_{\sigma-\mathbf{q}}}{\bar{\rho}_{\sigma}}+\frac{1}{36} q^{2} \frac{\rho_{\sigma-\mathbf{q}}}{\bar{\rho}_{\sigma}}\right]+g \rho_{\bar{\sigma}-\mathbf{q}}=0 \\
\frac{\partial \Omega}{\partial \bar{\rho}_{\sigma}}=\frac{\hbar^{2}}{2 m_{\sigma}}\left(6 \pi^{2} \bar{\rho}_{\sigma}\right)^{\frac{2}{3}}-\mu_{\sigma}+g \bar{\rho}_{\bar{\sigma}}=0
\end{gathered}
$$

Assuming $6 \pi^{2} \bar{\rho}_{\sigma}=\bar{k}_{\sigma}^{3}$ (note that in the presence of interactions, the average density and Fermi momentum, which we denote here by $\bar{\rho}_{\sigma}$ and $\bar{k}_{\sigma}$ respectively, are different from their non-interacting values), the above equations are simplified to:

$$
\begin{gathered}
\left(1+\frac{q^{2}}{24 \bar{k}_{\sigma}^{2}}\right) \rho_{\sigma \mathbf{q}}+2\left(\frac{\bar{k}_{\sigma} a}{\pi}\right) \rho_{\bar{\sigma} \mathbf{q}}=0 \\
\frac{\hbar^{2} \bar{k}_{\sigma}^{2}}{2 m}+\frac{4}{3}\left(\frac{\bar{k}_{\bar{\sigma}} a}{\pi}\right) \frac{\hbar^{2} \bar{k}_{\bar{\sigma}}^{2}}{2 m}=\frac{\hbar^{2} K_{F \sigma}^{2}}{2 m}=\mu_{\sigma}
\end{gathered}
$$

It is clear from the above equations that if $a=0$ then $\rho_{\sigma \mathbf{q}}=0$ is a solution (uniform density if no coupling). For $a>0$, we have $\rho_{\sigma \mathbf{q}}$ and $\rho_{\bar{\sigma} \mathbf{q}}$ of opposite signs for all $\mathbf{q}$. This means that there is phase segregation for repulsive couplings. Furthermore, if $a<0$, there will be density modulation in the small $\mathbf{q}$ limit (the functional we considered is valid in the long wavelength limit). We shall return to this point in section III where the dynamics are treated.

One can also note that the transition points of Eq. 16 and previously studied Eq. 6 are the same (in the $\omega=0$ and $\mu_{1}=\mu_{2}$ case), since they are derived from the same functional. Indeed from the positive-definiteness of the functional $\Omega$ in this representation, one obtains that the transition occurs for $\bar{k}_{\sigma} a / \pi=1 / 2$. Inserting this critical value into Eq. 16, one finds the relation between the non-interacting Fermi wavevector $K_{F}$ and the interacting one $\bar{k}_{\sigma}$ at the transition point: $K_{F}=\bar{k}_{\sigma} \sqrt{5 / 3}$ which then implies

$$
c=K_{F} a / \pi=\frac{1}{2} \sqrt{\frac{5}{3}} \approx 0.645,
$$

which is exactly the same value as obtained from the numerical result of the previous section.

\section{E. General case: Monte Carlo results}

The density distribution that extremizes the energy functional in Eq. (1) can be obtained by a Monte Carlo simulation with a weighting factor $\exp (-E / T)$ for a parameter $\mathrm{T}$ that is sufficiently low. This is basically the simulated annealing method and has been exploited successfully in earlier treatment 6 of the corresponding Bose system described by a Gross-Pitaevski functional.

We approximate the volume integral of the energy functional by a discrete sum. Using the scaled radius $\bar{r}$, we sample a lattice inside a sphere of diameter $2 R$ consisting of 40 sites along the diameter, making a total of 33398 sites. The derivative term is approximated by a finite difference. For simplicity, we show here results for the case when the two components have the same mass.

We first show in Fig. 2 the density profile of component 1 as a function of $\mathrm{x}$ and $\mathrm{y}$ for $\mathrm{z}=0$ for the weak coupling case with no phase segregation. The values of different parameters were chosen to be $\omega=135 \times 2 \pi \mathrm{rad} / \mathrm{sec}, a=135 a_{\mathrm{Bohr}}$, $\lambda=0.14$, and $N_{1}=N_{2}=10^{6}\left(\mu_{1}=\mu_{2}=1.626 \times 10^{-29} \mathrm{~J}\right)$; roughly corresponding to the experimental parameters of the ${ }^{40} K$ system 10 . In these experiments, we estimate $c=K_{F} a / \pi=0.032, R_{F}=26 \mu$ m, and $\mathcal{G}=0.042$. The density profile for component 2 is the same and hence is not shown.

In the limit of strong interaction, phase segregation starts, and as mentioned earlier, the system can now also break cylindrical symmetry. This happens when $K_{F}$ a is large enough, which in turn can be achieved with only large $K_{F}$, only large $a$, or both. To illustrate this, we show in Fig. 3 the density profiles for components 1 and 2 for the case of only large $a$ with $a=30000 a_{\text {Bohr }}, \mu_{1}=\mu_{2}=1.86 \times 10^{-30} \mathrm{~J}$, and $\omega=300 \mathrm{rad} / \mathrm{sec}$. In this case, $c=K_{F} a / \pi=2.39$, $R_{F}=25 \mu \mathrm{m}$, and $\mathcal{G}=3.19$. 
For the case of both large $K_{F}$ and $a$, we show in Fig. 4 the density profiles for $a=3000 a_{\mathrm{Bohr}}, \mu_{1}=\mu_{2}=$ $2.762 \times 10^{-29} \mathrm{~J}$, and $\omega=2000 \mathrm{rad} / \mathrm{sec}$. This corresponds to $c=0.92, R_{F}=14.3 \mu \mathrm{m}$, and $\mathcal{G}=1.23$. The difference in the densities of the two components shows that the largest change occurs near the center where the density is maximum.

It is to be further noted that for this case, the density distribution is still quite cylindrical but there is a slight asymmetry, as we can see from the graph of the difference. This asymmetry becomes more pronounced as the interaction is increased further. In Fig. 5 we have shown the results of simulations with larger $a$. The density profiles were calculated for $a=4160 a_{\text {Bohr }}, \mu_{1}=\mu_{2}=1.626 \times 10^{-29} \mathrm{~J}, \omega=6000 \mathrm{rad} / \mathrm{sec}$. This corresponds to $c=0.98, R_{F}=3.67 \mu \mathrm{m}$, and $\mathcal{G}=1.31$.

As discussed earlier, phase separation can also occur when $N_{1}>>N_{2}$. As an illustration, we show in Fig. 6 the density profiles for components 1 and 2 for the case $a=104 a_{\mathrm{Bohr}}, \mu_{1}=2.6016 \times 10^{-26} \mathrm{~J}, \mu_{2}=4.336 \times 10^{-26} \mathrm{~J}$ and $\omega=1600000 \mathrm{rad} / \mathrm{sec}$.

The density of component 2 is small and therefore, its noise is also substantially higher. One can clearly see the density depletion of component 2 at the center.

\section{DYNAMICS}

We next turn our attention to the issue of dynamics. For the classical and boson spinodal decompositions, the fastest unstable mode occurs at a finite wave vector. We ask if a similar situation occurs for the fermion case. We found that the fastest unstable mode occurs at wavevector $q=0$ at the onset of instability. For stronger coupling, many modes with $q \sim K_{F}$ decay with comparable time scales. We now describe the details of this linear stability analysis.

The energy functional (Eq. (1)) which was approximated with a local kinetic energy depending on the density and its derivatives is only good in the long wavelength limit. Due to this approximation, we found that the instability has a local character and occurs first in regions of high density. Here we will perform a linear instability analysis in the random phase approximation (RPA) to improve upon this local picture. The linear susceptibility $\chi$ is defined as the response of the particle density to an external potential $V$ which could also be $\sigma$-dependent:

$$
\delta \rho_{\sigma}(\mathbf{r})=\sum_{\sigma^{\prime}=1,2} \int d \mathbf{r}^{\prime} \chi_{\sigma \sigma^{\prime}}\left(\mathbf{r}, \mathbf{r}^{\prime}\right) V_{\sigma^{\prime}}^{t o t}\left(\mathbf{r}^{\prime}\right)
$$

Here $V^{t o t}$ is the total self-consistent field and is the sum of the external field and that due to the interaction: $V_{\sigma}^{\text {tot }}=V_{\sigma}+g \delta \rho_{\bar{\sigma}}$. The bare response $\chi_{\sigma \sigma}$ can be obtained from the usual Lindhard expression 18 . Since there is no term in the Hamiltonian that interchanges the species 1 and 2, off-diagonal terms of the susceptibility are zero $\left(\chi_{12}=\chi_{21}=0\right)$. Taking the above into consideration, Eq. (18) can be written in the following matrix form: $\delta \rho=\chi(V+G \delta \rho)$, leading to $\delta \rho=[1-\chi G]^{-1} \chi V$, where the $2 \times 2$ matrix $G$ has 0 as its diagonal elements and $g$ as its off-diagonal elements, and $\chi$ is diagonal. Consequently, an instability will occur when the following determinant becomes zero:

$$
\operatorname{Det}|1-\chi G|=1-g^{2} \chi_{11} \chi_{22}=0
$$

In the case where the densities are equal, $\chi_{11}=\chi_{22} \equiv \chi$, the two eigenmodes are calculated as:

$$
\begin{aligned}
& \delta \rho_{1}+\delta \rho_{2}=(1-\chi g)^{-1} \chi\left(V_{1}+V_{2}\right) \\
& \delta \rho_{1}-\delta \rho_{2}=(1+\chi g)^{-1} \chi\left(V_{1}-V_{2}\right) .
\end{aligned}
$$

The first mode corresponds to a density fluctuation, and the second mode $\delta \rho_{1}-\delta \rho_{2}$ represents the phase separation instability in which we are interested. The response corresponding to this mode is given by $\epsilon(q, w)=[1+g \chi(q, w)]$. The instability decay time $\nu^{-1}$ is determined from the formula $\epsilon(q, i \nu)=0$, since, in this case, any infinitesimal external potential will lead to a large change in the density. There exists a $q=q_{0}$ such that $\nu\left(q_{0}\right)$ is largest. This determines the spinodal wavevector of the fermionic system as it indicates the mode with fastest growth. In what follows, we will be treating the constant external potential problem where the Fermi momentum is $\bar{k}$. For the confined case, one an consider $\bar{k}$ to be a local function related to the density by $\bar{k}(\mathbf{r})=\left[6 \pi^{2} \rho(\mathbf{r})\right]^{1 / 3}$. From the Lindhard expression 18 for $\chi$ (real frequencies), we obtain, after correcting for a spin degeneracy factor of 2 , the corresponding dimensionless response $\bar{\chi}=-4 \pi^{2} \hbar^{2} \chi(q, i \nu) / m \bar{k}$ for imaginary frequencies:

$$
\bar{\chi}(q, i \nu)=1+\frac{1}{2 q}\left(1+(\nu / q)^{2}-(q / 2)^{2}\right) \log \left[\frac{(1+q / 2)^{2}+(\nu / q)^{2}}{(1-q / 2)^{2}+(\nu / q)^{2}}\right]
$$




$$
-\frac{\nu}{q}\left(\tan ^{-1}\left[\frac{\nu / q}{(1-q / 2)}\right]+\tan ^{-1}\left[\frac{\nu / q}{(1+q / 2)}\right]\right)
$$

Here $q$ is in units of $\bar{k}$ and $\nu$, in units of $\hbar / 2 \bar{E}=m / \hbar \bar{k}^{2}$. The three-dimensional plot of $\bar{\chi}$ as a function of $q$ and $\nu$ is shown in Fig. 7 .

The equation $\epsilon(q, w)=[1 \pm g \chi(q, w)]=0$ implies that the instability points for phase segregation with a repulsive interaction $(g>0)$ and that of density modulation with an attractive interaction are exactly the same within RPA. This is also in agreement with the analysis of section II E where it was shown that "magnetic" instability occurs for repulsive interactions, and "density wave" instability may occur for attractive interactions. Although the susceptibility can be both negative or positive, for a coupling of fixed sign, one should only consider the physically correct situation. In our case, for positive $g$, only the "magnetic" instability, i.e. $\chi=-1 / g$ should be considered.

Now since $g \chi=-\bar{\chi} \bar{k} a / \pi$, the instability condition implies $\bar{c} \bar{\chi}=1$ where $\bar{c}=\bar{k} a / \pi$. The maximum of $\bar{\chi}$ is obtained for $q \rightarrow 0$ and $\omega \rightarrow 0$ where it tends to 2 . From this result, we arrive at the conclusion that there is no solution to $\epsilon(q, i \nu)=0$ for $\bar{c}<0.5$ and no instability develops. For larger values of $\bar{c}$, the plane $z=1 / \bar{c}$ intersects the surface of $\bar{\chi}$ on a curve which is displayed in Fig. 8. The inverse decay time $\omega$ as a function of the wavevector in units of $\bar{k}$ is shown in this figure. As can be seen, the fastest unstable mode occurs at wavevector $q=0$ and $\omega=0$ at the onset of the instability $(\bar{c}=0.5)$ in agreement with Eq. 17 previously derived. Indeed the instability calculation derived in the previous section focused on the long wavelength aspect of the problem.

For stronger couplings, many modes with $q \approx \bar{k}$ decay with comparable time scales of the order of $\hbar / E_{F}$, but those with shortest timescales (i.e. largest $\omega$ ) prevail.

In the really strong interaction limit, further phase separation can take place either via tunnelling 192.20 or via quantum motion of the domain walls. We hope to investigate this further in the future.

The behavior of the wavevector of instability is similar to that of the classical spinodal decomposition, which we briefly recapitulate here. The current $J$ can be related to the free energy $F$ by Fick's law: $J=c \nabla F$ for some constant c. After the onset of instability, $F=\left(-A+B q^{2}\right) \delta \rho_{q}$. As one goes from the onset of instability, $A$ starts to become non-zero. In addition, there is the particle conservation equation $-\partial_{t} \rho=\nabla \cdot J$. Combining the above two equations, we obtain $i \omega \delta \rho_{q}=c q^{2}\left(-A+B q^{2}\right) \delta \rho_{q}$. The fastest mode occurs at a wavevector $q_{c}=\sqrt{A / 2 B}$. Thus at the onset of instability, $q_{c}=0 . q_{c}$ becomes larger as one goes away from the instability point.

\section{CONCLUSION}

In conclusion we have investigated the statics and dynamics of the spatial phase segregation process of a mixture of fermion atoms in a harmonic trap using the density functional theory and the random phase approximation. As the coupling starts to increase, even with the same chemical potential, equilibrium distribution with unequal densities starts to appear, which quite often do not exhibit axially symmetric correlations. Similar to the classical and Bose spinodal decomposition cases, the fastest mode for the initial phase segregation occurs at a finite wave-vector. The condition of instability corresponds to a large interaction, which may be achieved experimentally with the atoms close to a Feshbach resonance.

The instability calculation for the phase segregation phenomena discussed here is related to the instability calculation for the antiferromagnetic transition of the electron gas. In the electron gas, this is enhanced when there is nesting of Fermi surface such as in $\mathrm{Cr}$ or in one dimensional materials. The transition always stops after the $2 K_{F}$ instability due to the long range nature of the Coulomb interaction, and no further "segregation" takes place.

An interesting situation is the one dimensional trap as it would exhibit a much stronger instability. In mean field, the one dimensional density difference response function $\epsilon\left(2 K_{F}\right)=1 /\left[1+K_{F} a \log \left(T / E_{F}\right)\right]$ is $\operatorname{logarithmically}$ divergent at zero temperature. The transition temperature occurs af $T_{c}=E_{F} e^{-1 / K_{F} a}$. One dimensional trap, which can be realized for small values of $\lambda$, has been extensively studied 310 and we expect a higher tendency towards phase segregation in that case as well.

\section{ACKNOWLEDGMENTS}

S.T. Chui is partly supported by NASA under contract no. NAG8-1427. He, KE and VK thank the Institute for Materials Research for the kind hospitality, where the main body of this work was completed. 
${ }^{\dagger}$ Permanent address: Sharif University of Technology, and Institute for Physics and Mathematics, Tehran, Iran.

* Permanent address: Bartol Research Institute, University of Delaware, Newark, DE 19716, USA.

** Permanent address: Dr. Vijay Kumar Foundation, 45 Bazaar Street, K.K. Nagar (West), Chennai - 600078 , India.

${ }^{1}$ C. J. Myatt, E. A. Burt, R. W. Ghrist, E. A. Cornell, and C. E. Wieman, Phys. Rev. Lett. 78, 586 (1997).

${ }^{2}$ D. S. Hall, M. R. Matthews, J. R. Ensher, C. E. Wieman, and E. A. Cornell, Phys. Rev. Lett. 81, 1539 (1998).

${ }^{3}$ J. Stenger, S. Inouye, D. M. Stamper-Kurn, H. J. Miesner, A. P. Chikkatur, and W. Ketterle, Nature 396, 345 (1998).

${ }^{4}$ C. K. Law, H. Pu, N. P. Bigelow, and J. H. Eberly, Phys. Rev. Lett. 79, 3105 (1997); and references therein.

${ }^{5}$ T. L. Ho, Phys. Rev. Lett. 81, 742 (1998).

${ }^{6}$ S. T. Chui and P. Ao, Phys. Rev. A59, 1473 (1999).

${ }^{7}$ Y. Iwama and M. Takeuchi, Trans. Jap. Inst. Met. 15, 371 (1974); ibid. 17, 481 (1976).

${ }^{8}$ J. W. Cahn, Tans. Met. Soc. AIME 242, 166 (1968); J. S. Langer, in Solids Far From Equilibrium, ed. C. Godrèche (Cambridge University Press, Cambridge, 1992).

${ }^{9}$ P. Ao and S. T. Chui, To appear in J. Phys. Conds. Matt. and Physica.

10 B. DeMarco and D. S. Jin, Science 285, 1703 (1999).

${ }^{11}$ K. M. O'Hara, S. R. Granade, M. E. Gehm, T. A. Savard, S. Bali, C. Freed, and J.E. Thomas, Phys. Rev. Lett. 82, 4204 (1999).

${ }^{12}$ W. F. Brinkman, T. M. Rice, P. W. Anderson and S. T. Chui, Phys. Rev. Lett. 28, 961 (1972).

13 D. A. Butts and D. S. Rokhsar, Phys. Rev. A55, 4346 (1997).

${ }^{14}$ G. M. Bruun and K. Burnett, Phys. Rev. A58, 2427 (1998).

${ }^{15}$ J. Schneider and H. Wallis, Phys. Rev. A57, 1253 (1998).

${ }^{16}$ M. Houbiers, R. Ferwerda, H. T. C. Stoof, W. I. McAlexander, C. A. Sackett, and R. G. Hulet, Phys. Rev. A56, 4864 (1997).

${ }^{17}$ P. Ao and S.T. Chui, Phys. Rev. A58, 4836 (1998).

18 A. Fetter and D. Walecka, Quantum Many-Body Systems, Mc Graw Hill (1971); G. D. Mahan, Many Particle Physics, Plenum (1990).

${ }^{19}$ I. Zapata, F. Sols, and A. J. Leggett, Phys. Rev. A57, R28 (1998).

${ }^{20}$ A. Smerzi, S. Fantoni, S. Giovanazzi, and S. R. Shenoy, Phys. Rev. Lett. 79, 4950 (1997).

FIG. 1. Top: Dimensionless density versus dimensionless radius $\bar{r} / R$ for $\mathcal{G}=1$. One of the asymmetric solutions (A2) is depleted at the center while the other one has a large concentration. For $\bar{r} / R$ larger than 0.51 both asymmetric solutions join the symmetric density profile. The sharp features around this point are due to the neglect of the gradient terms. Bottom: Universal curve of rescaled density $\mathcal{N}=n \mathcal{G}^{3}$ versus rescaled distance from the border $\mathcal{P}=\left(1-\bar{r}^{2} / R^{2}\right) \mathcal{G}^{2}$, valid for all coupling strengths $\mathcal{G}$. Note that $0<\mathcal{P}<1$, and for the symmetric case $\mathcal{N}_{\max }=0.43(\bar{r}=0$ or $\mathcal{P}=1)$.

FIG. 2. Snap shot of the density profile at $\mathrm{z}=0$ as a function of $\mathrm{x}$ and $\mathrm{y}$ in the weak coupling limit $c=0.032$.

FIG. 3. Snap shot of the density profile of components 1 (top) and 2 (bottom) at $\mathrm{z}=0$ as a function of $\mathrm{x}$ and $\mathrm{y}$ in the strong coupling limit $c=2.39, \omega=300 \mathrm{rad} / \mathrm{sec}$.

FIG. 4. Snap shot of the density profile of components 1 and 2 and their difference at $\mathrm{z}=0$ as a function of $\mathrm{x}$ and $\mathrm{y}$ in the strong coupling limit $(c=0.92, \omega=2000 \mathrm{rad} / \mathrm{sec})$.

FIG. 5. Snap shot of the density profiles of components 1 and 2 at $\mathrm{z}=0$ as a function of $\mathrm{x}$ and $\mathrm{y}$ in the strong coupling limit $(c=0.98, \omega=6000 \mathrm{rad} / \mathrm{sec})$.

FIG. 6. Snap shots of the density profiles at $\mathrm{z}=0$ as a function of $\mathrm{x}$ and $\mathrm{y}$ for $c_{1}=0.98, c_{2}=1.27, \omega=1600000 \mathrm{rad} / \mathrm{sec}$. Density 2 is depleted in the central region.

FIG. 7. Surface plot of the positive part of the reduced Lindhard susceptibility $(\bar{\chi})$ as a function of $q / \bar{k}$ and the imaginary frequency. 
FIG 8. Contour plots of the Imaginary frequency Lindhard susceptibility indicating the inyerse decay time for the phase segregation mode of wave vector $q$ for several values of the dimensionless coupling $1 / c=\pi / \bar{k} / a=0,0.3,0.65,1,1.45,1.75,1.9,1.98$ starting from the outermost line representing $\bar{\chi}=0$.

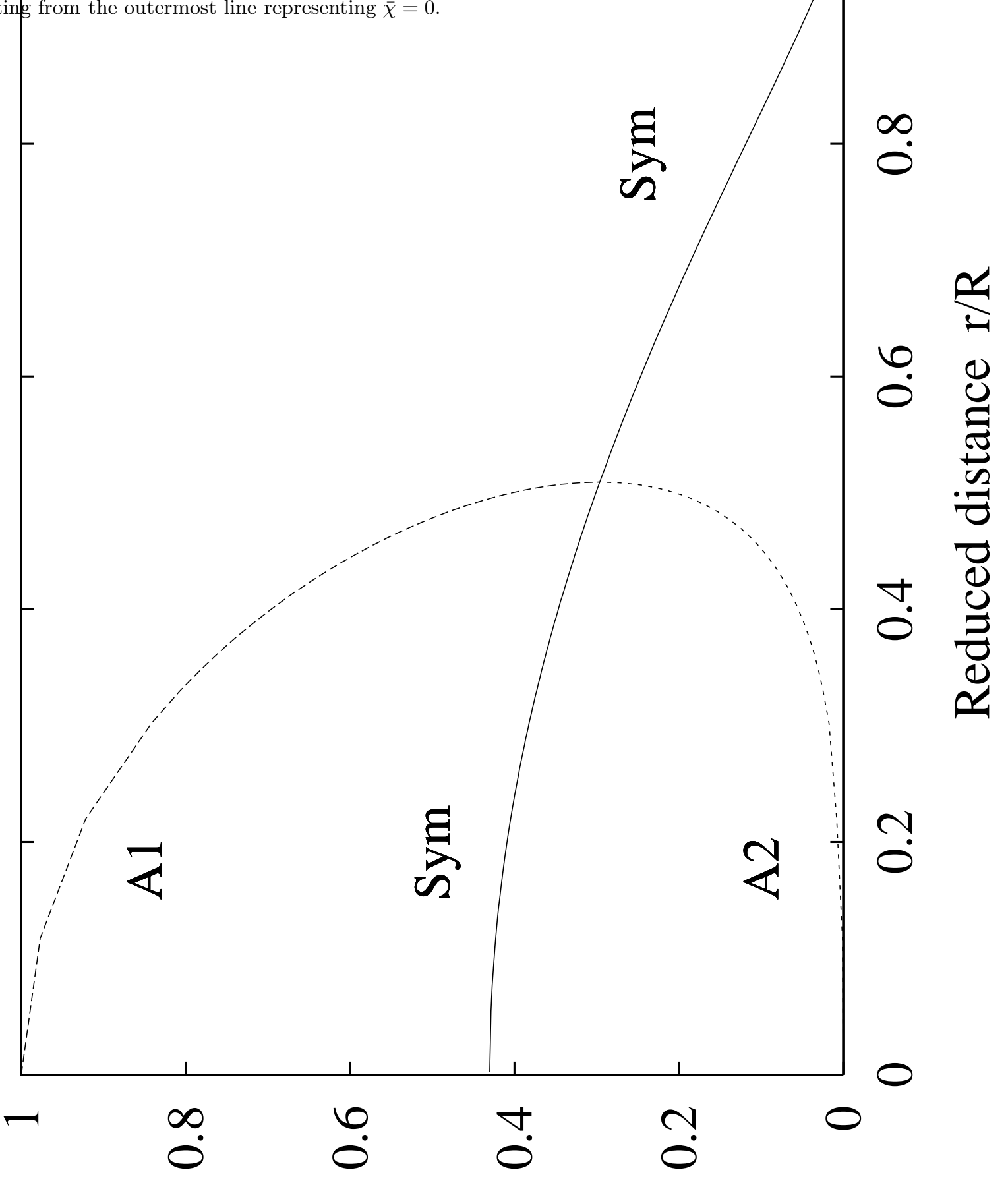

\section{(I)U Kł!ฺsuәp pəonpəy}

Fig. 1 (top), K. Esfarjani et al. 


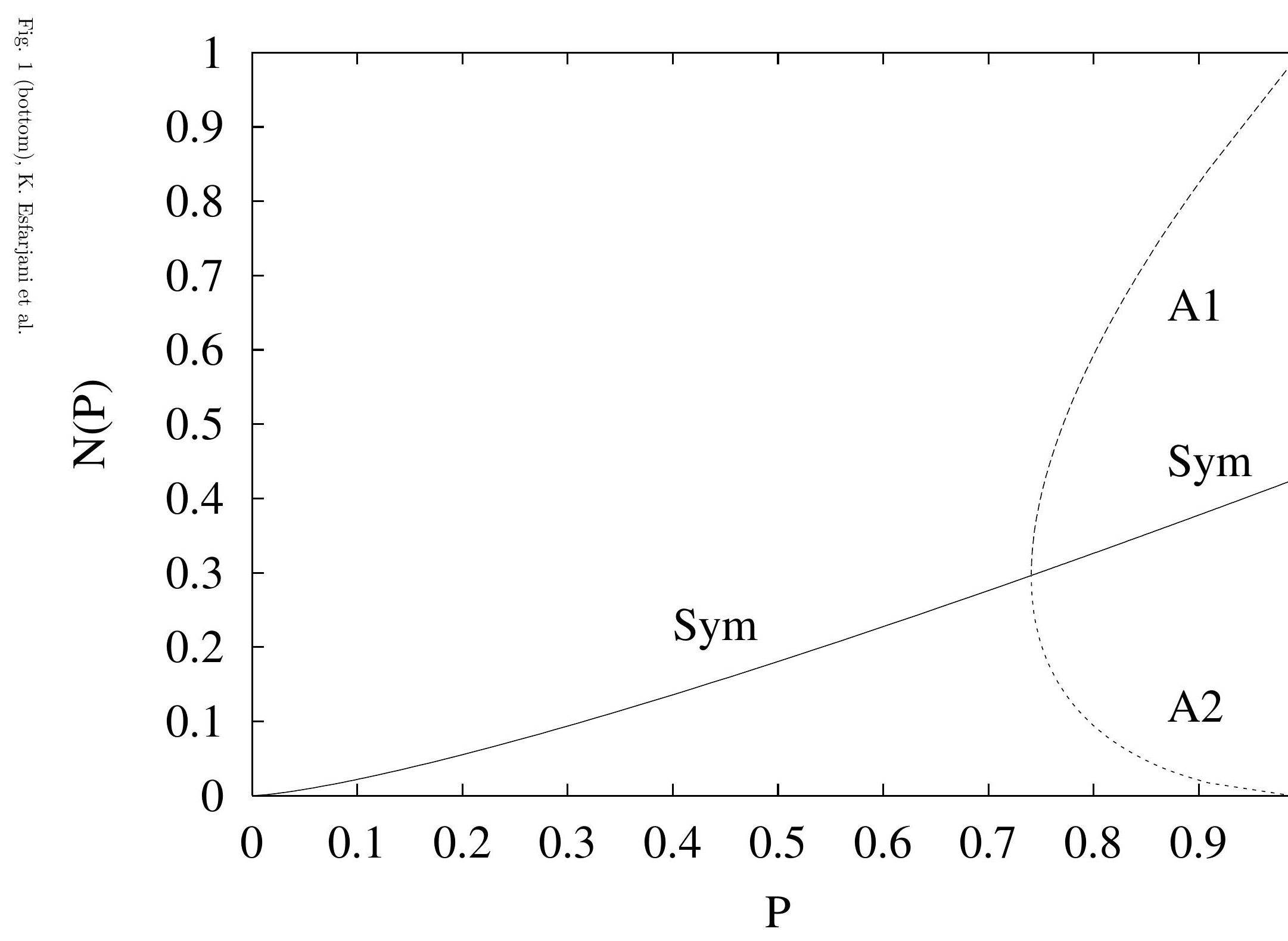




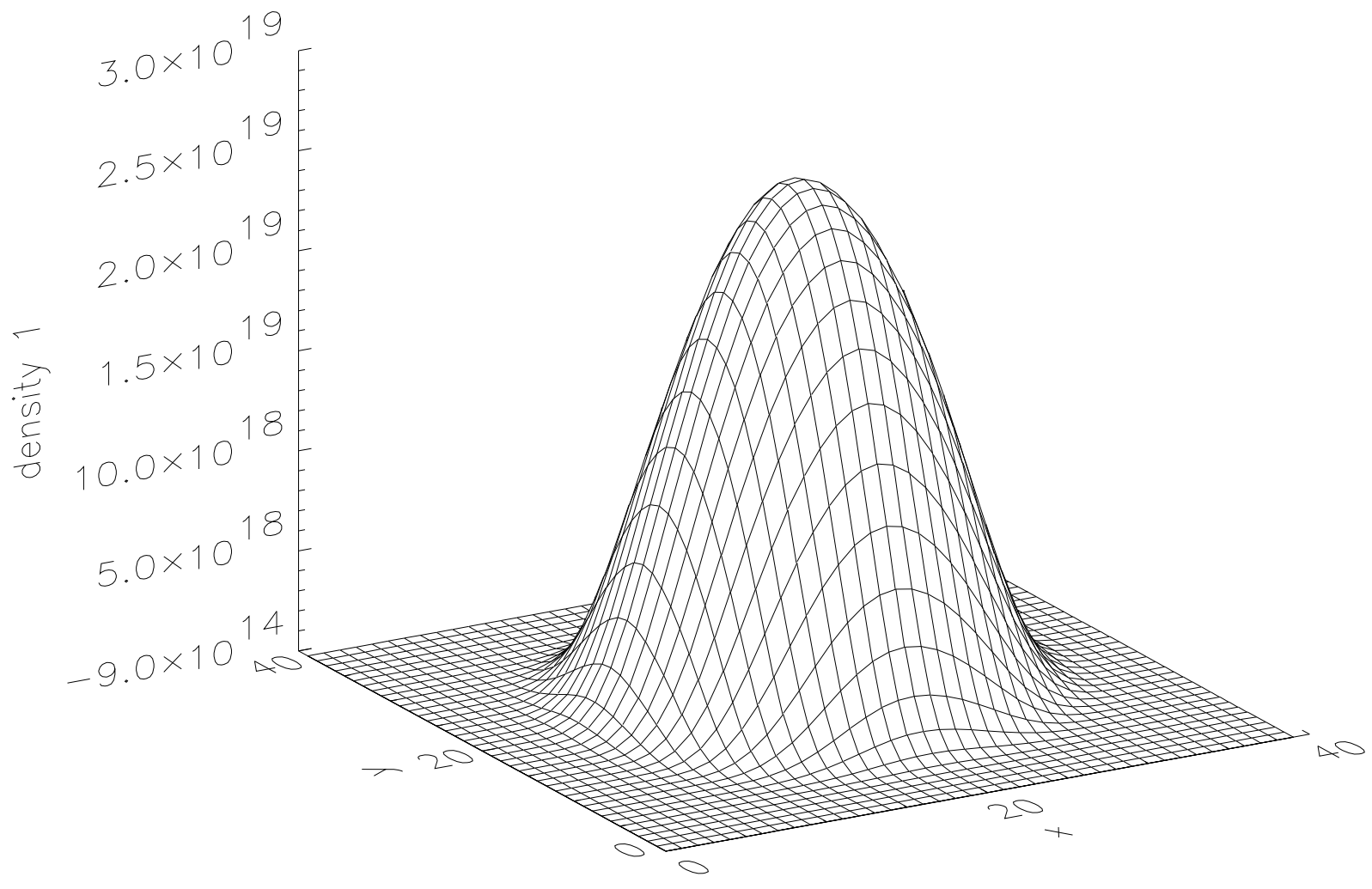

Fig. 2, K. Esfarjani et al. 


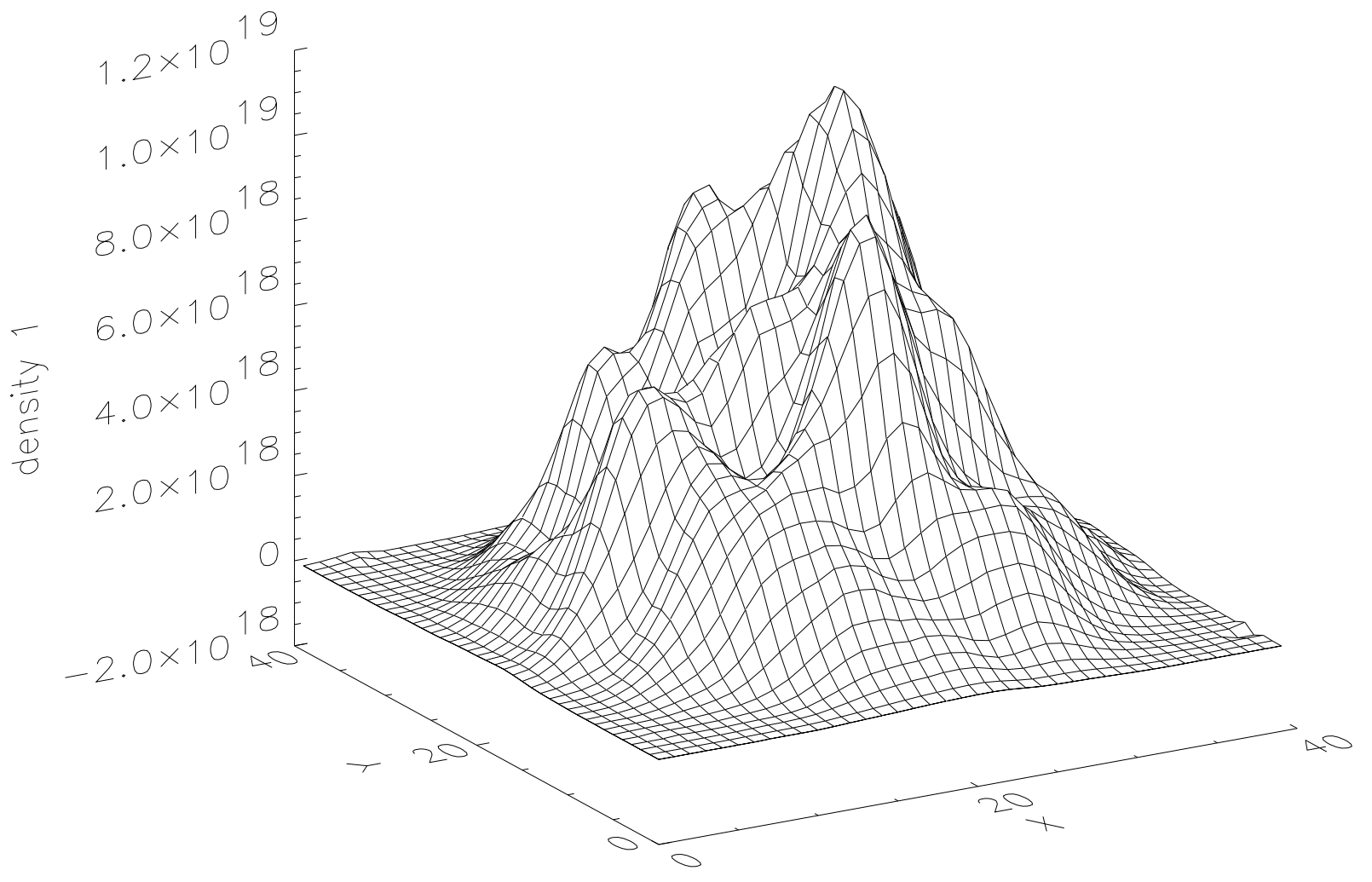

Fig. 3 (top:density 1), K. Esfarjani et al. 


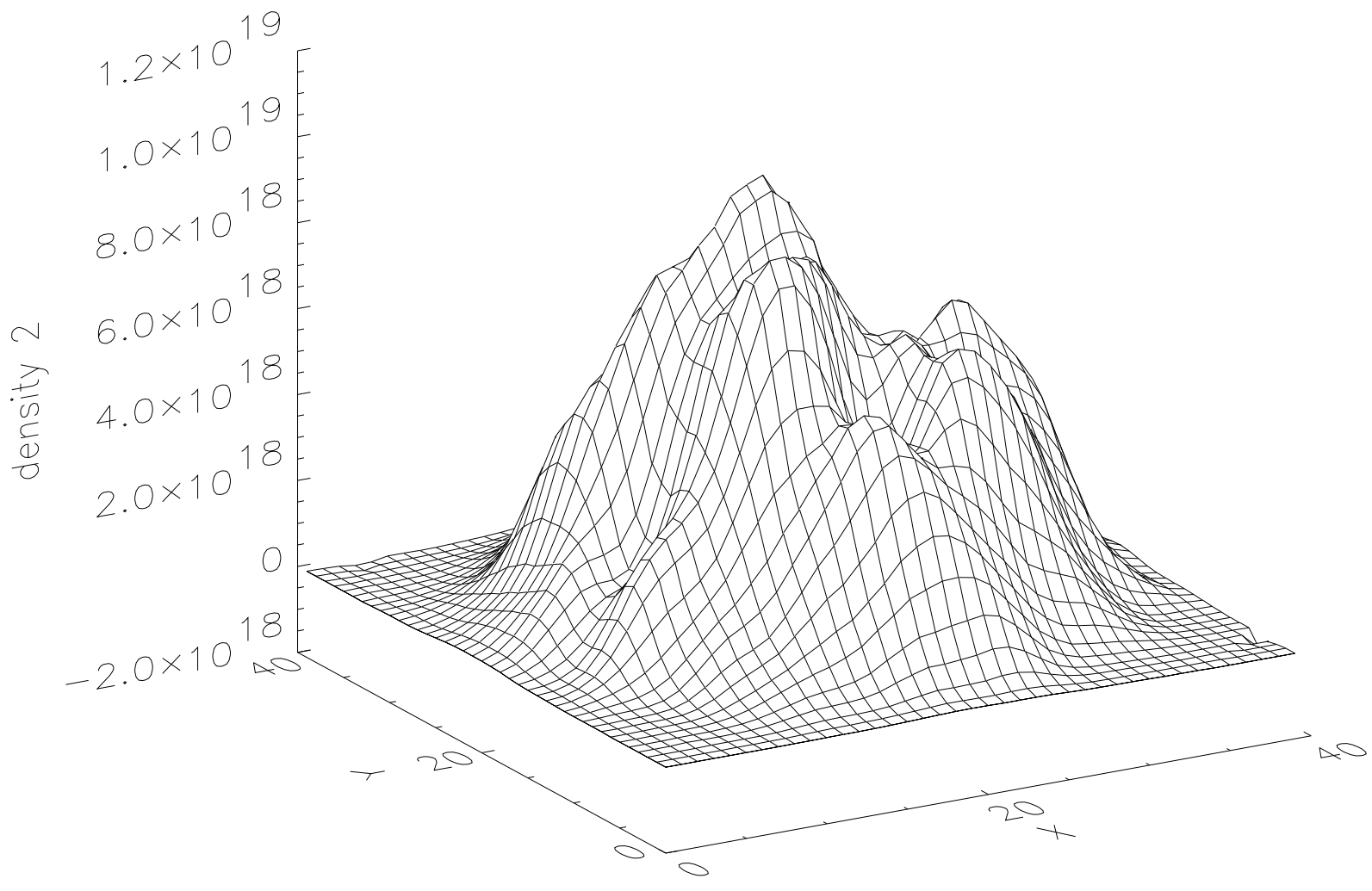

Fig. 3 (bottom:density 2), K. Esfarjani et al. 


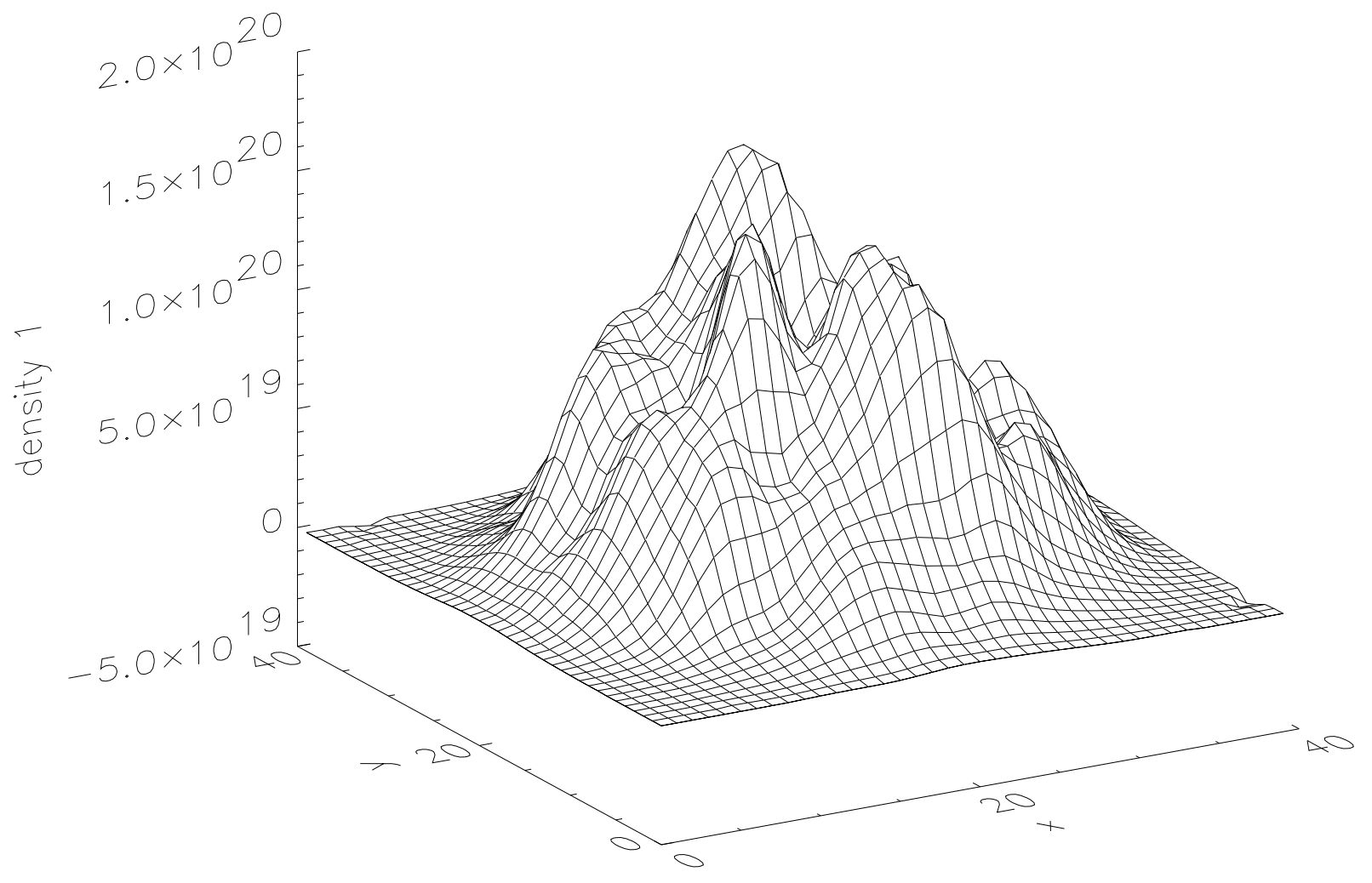

Fig. 4 (top:density 1), K. Esfarjani et al. 


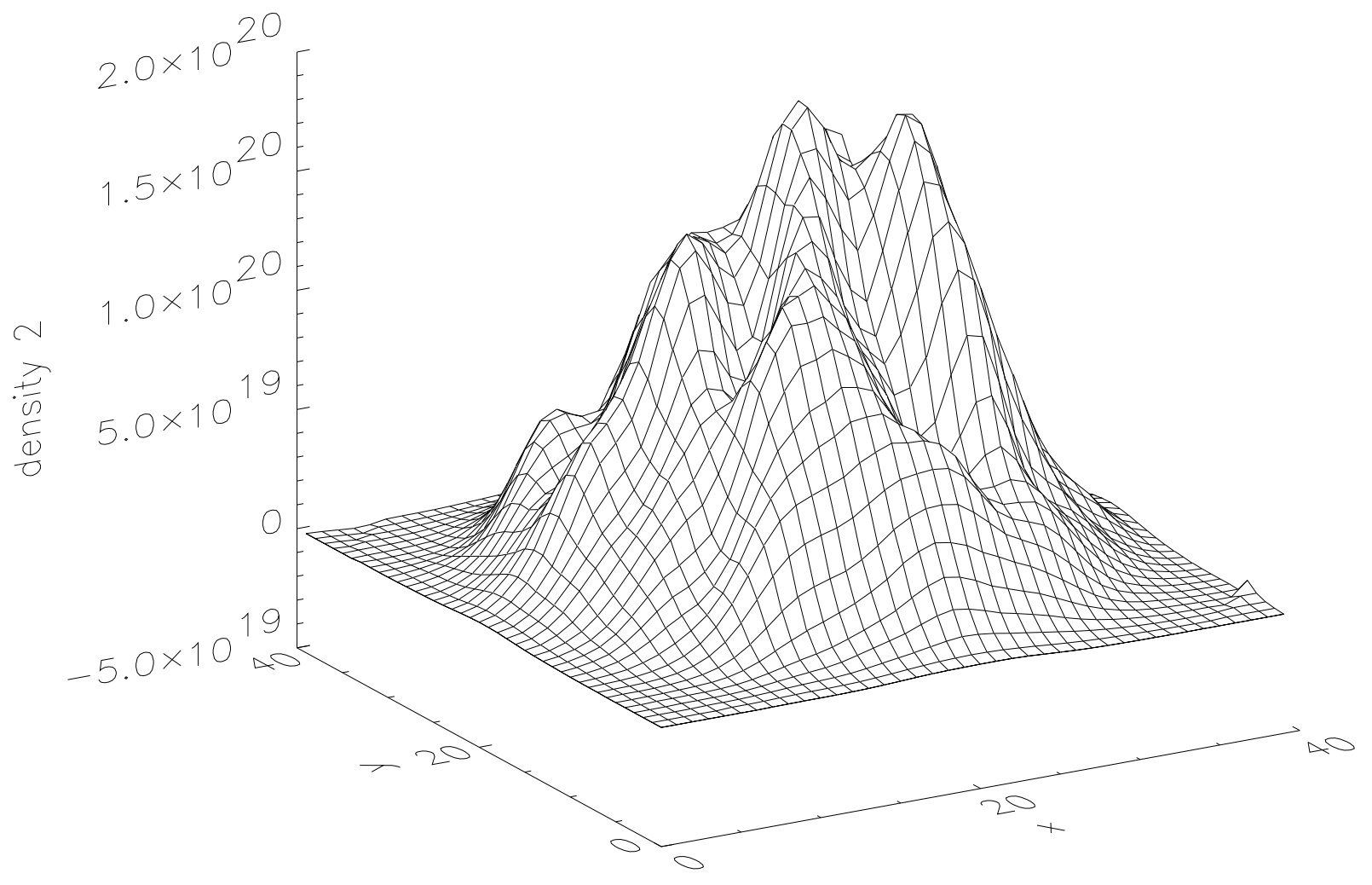

Fig. 4 (middle:density 2), K. Esfarjani et al. 


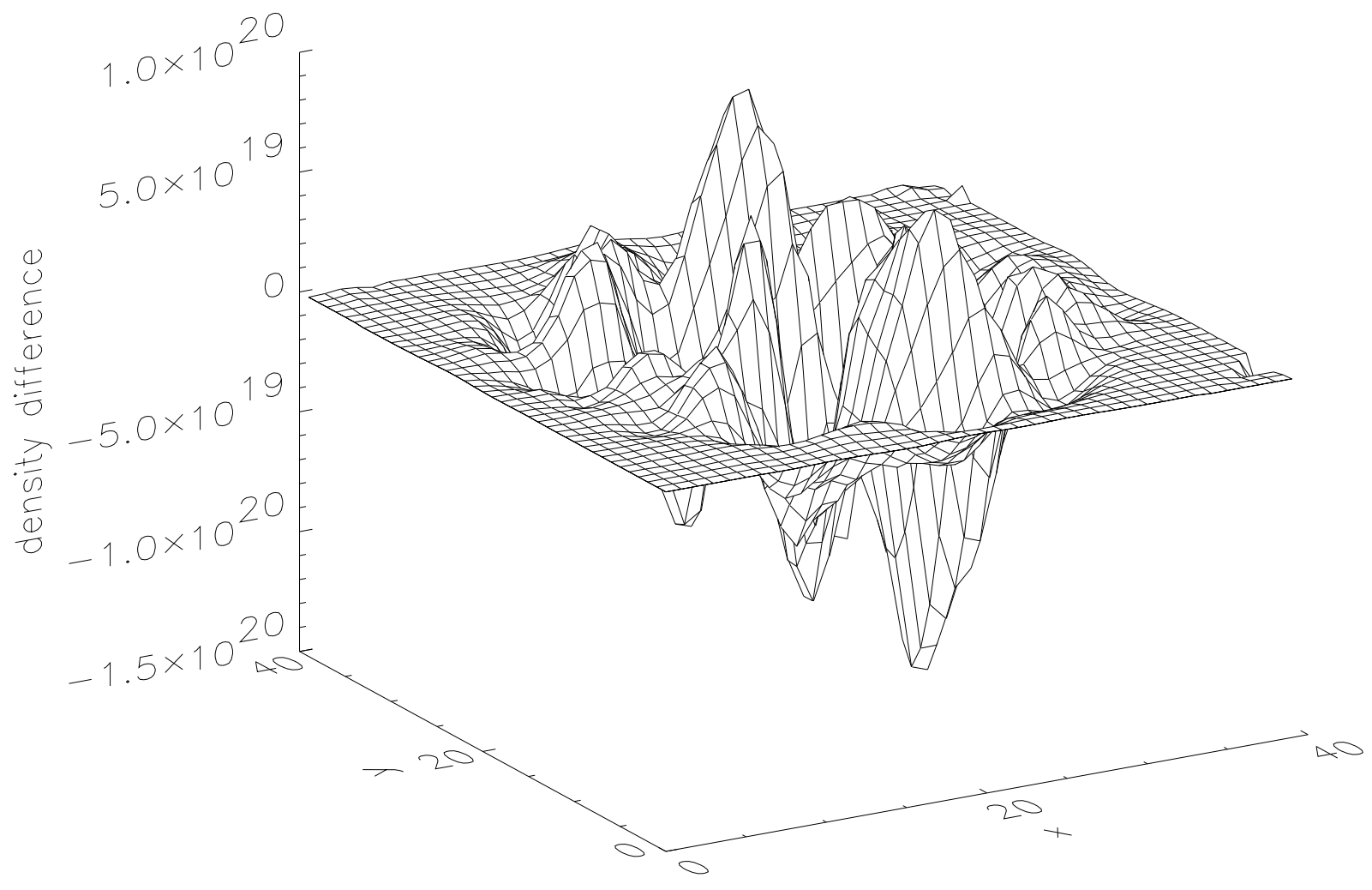

Fig. 4 (bottom:density difference), K. Esfarjani et al. 


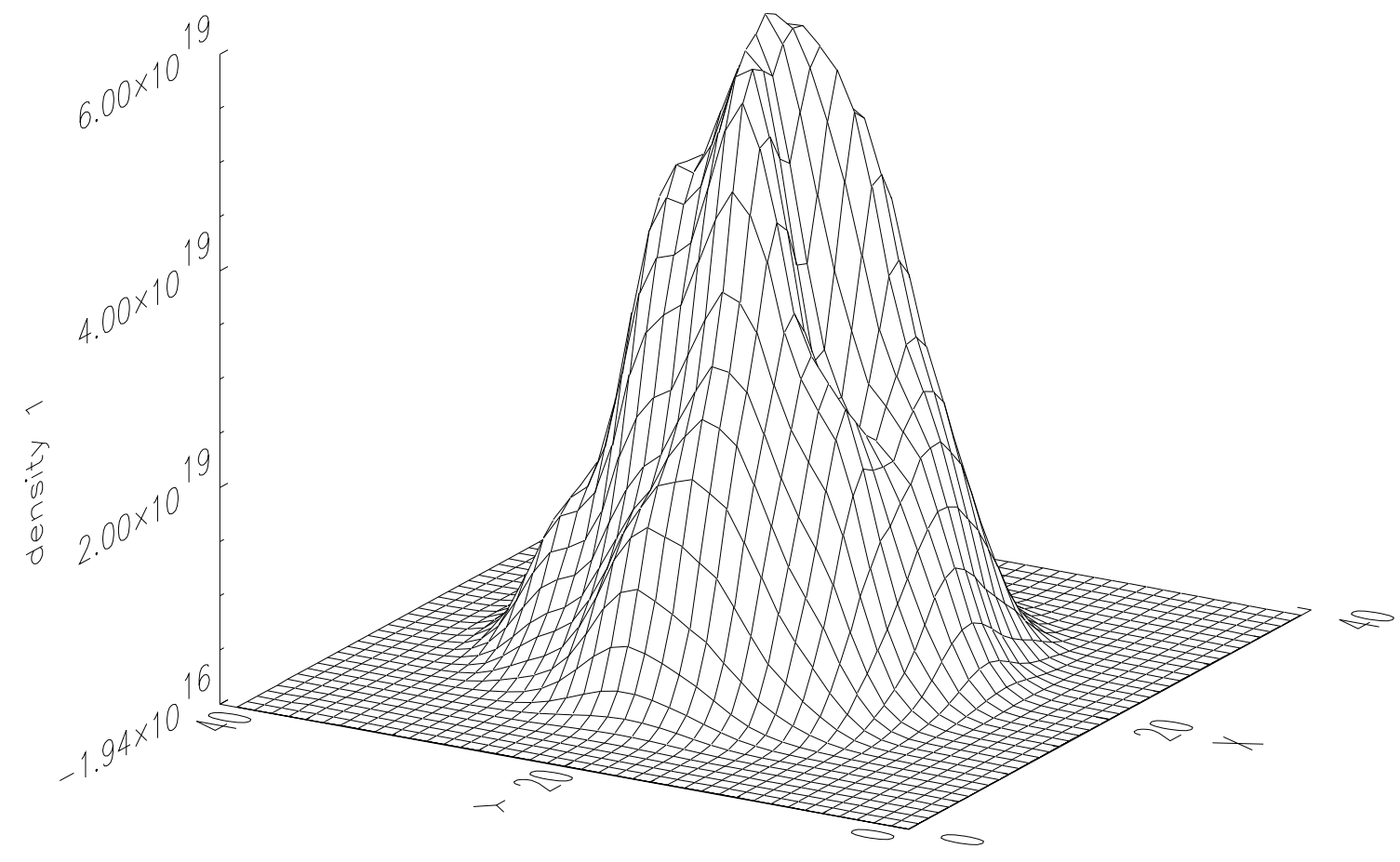

Fig. 5 (top:density 1), K. Esfarjani et al. 


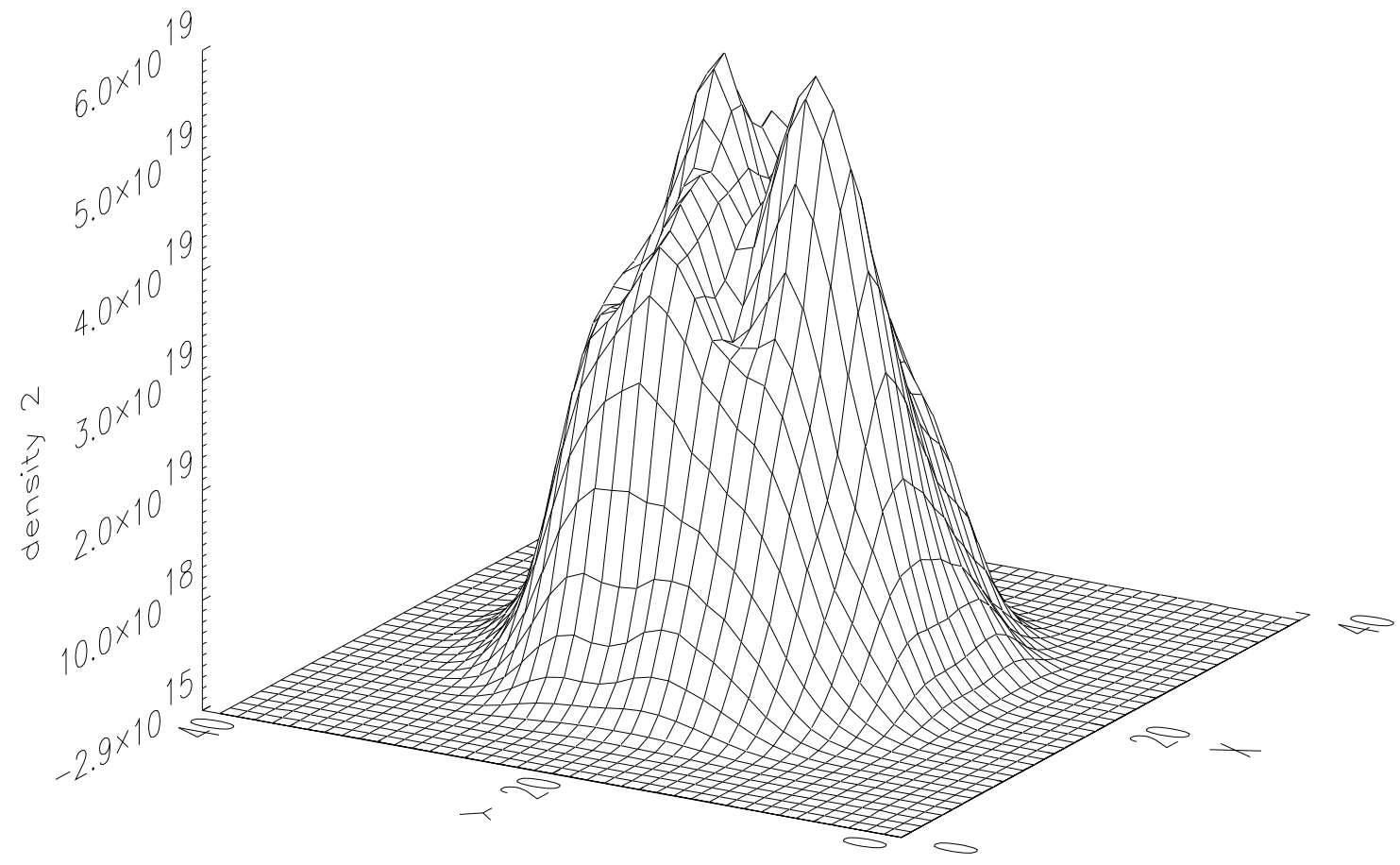

Fig. 5 (bottom:density 2), K. Esfarjani et al. 


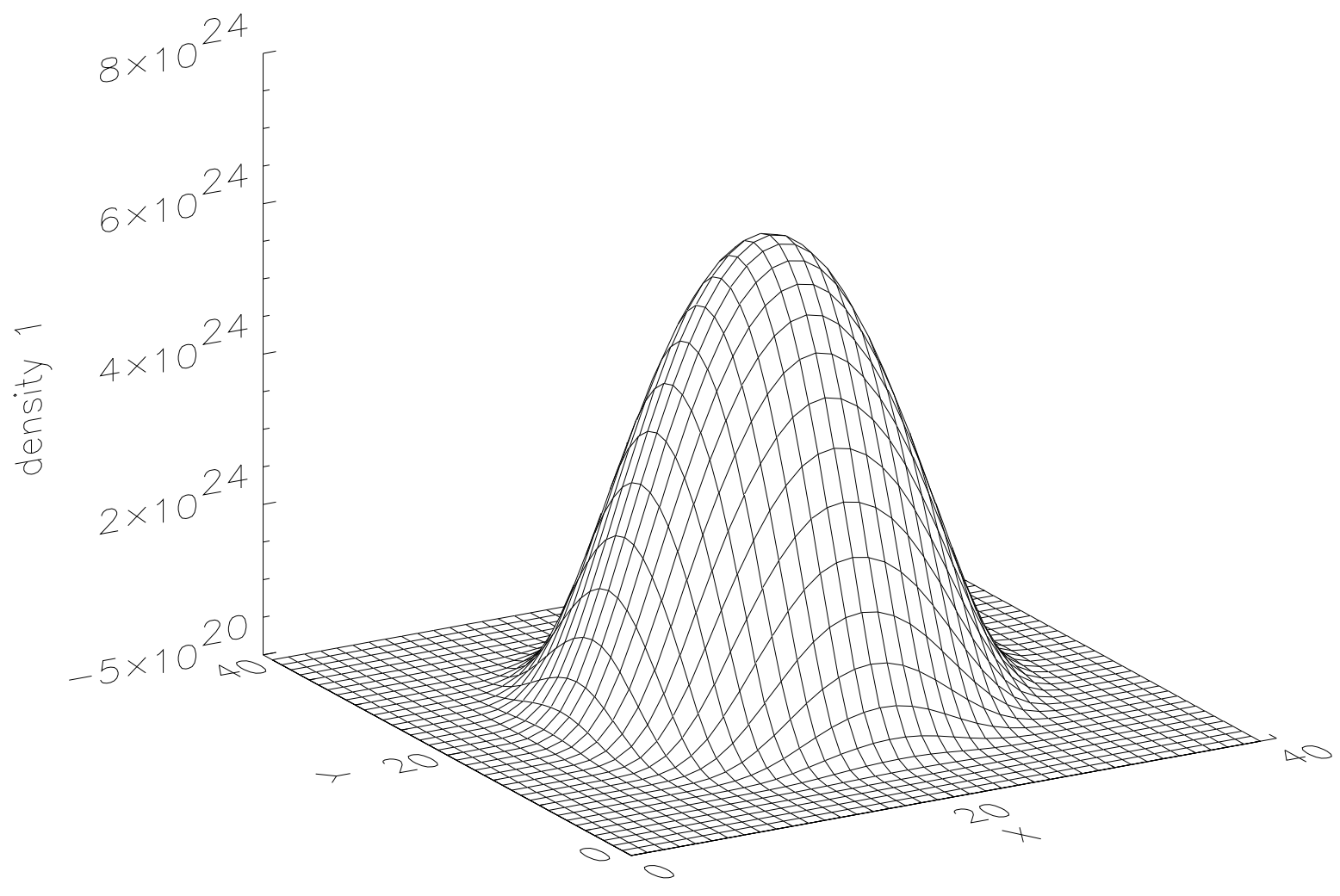

Fig. 6 (top:density 1), K. Esfarjani et al. 


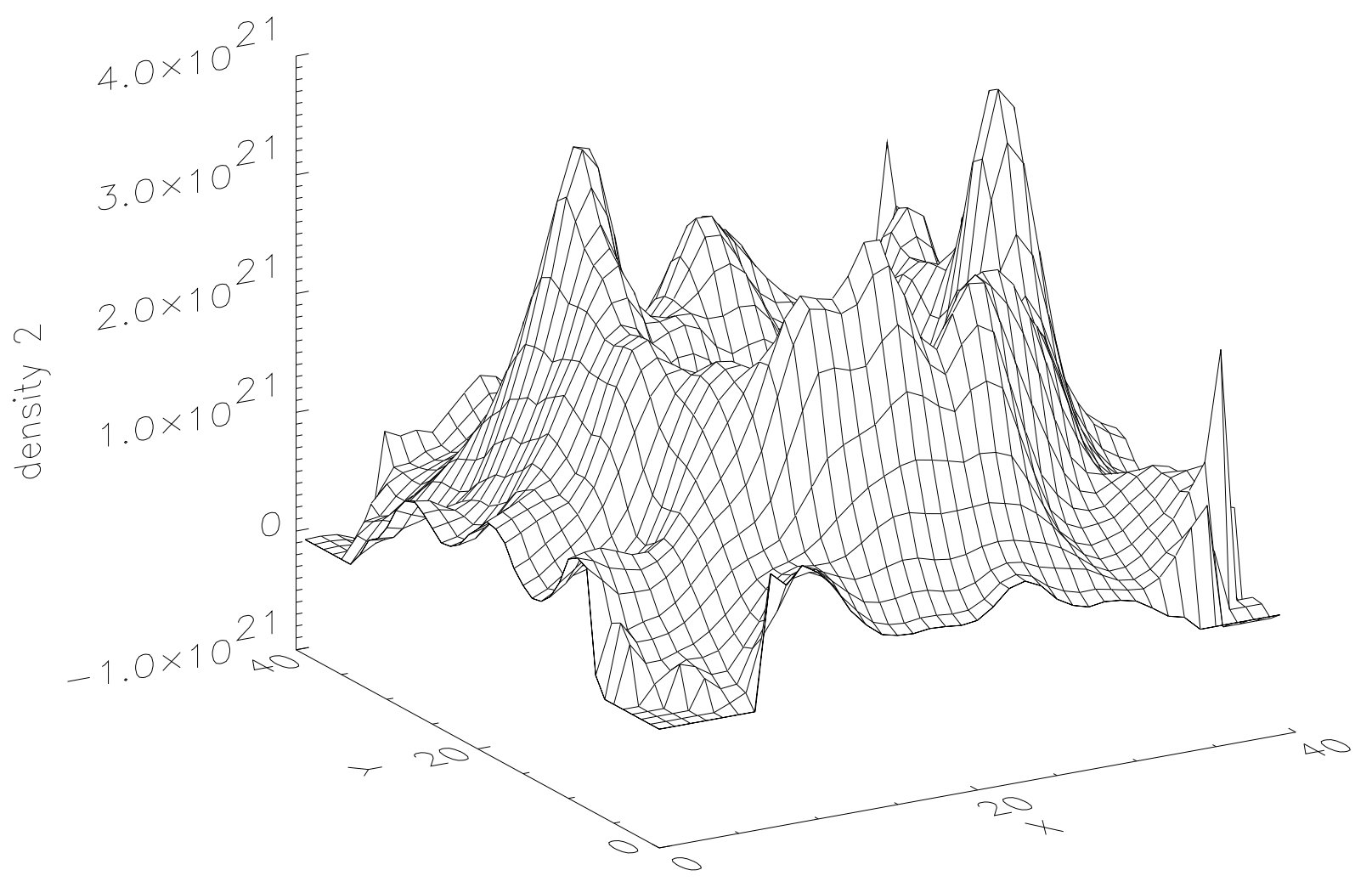

Fig. 6 (bottom:density 2), K. Esfarjani et al. 


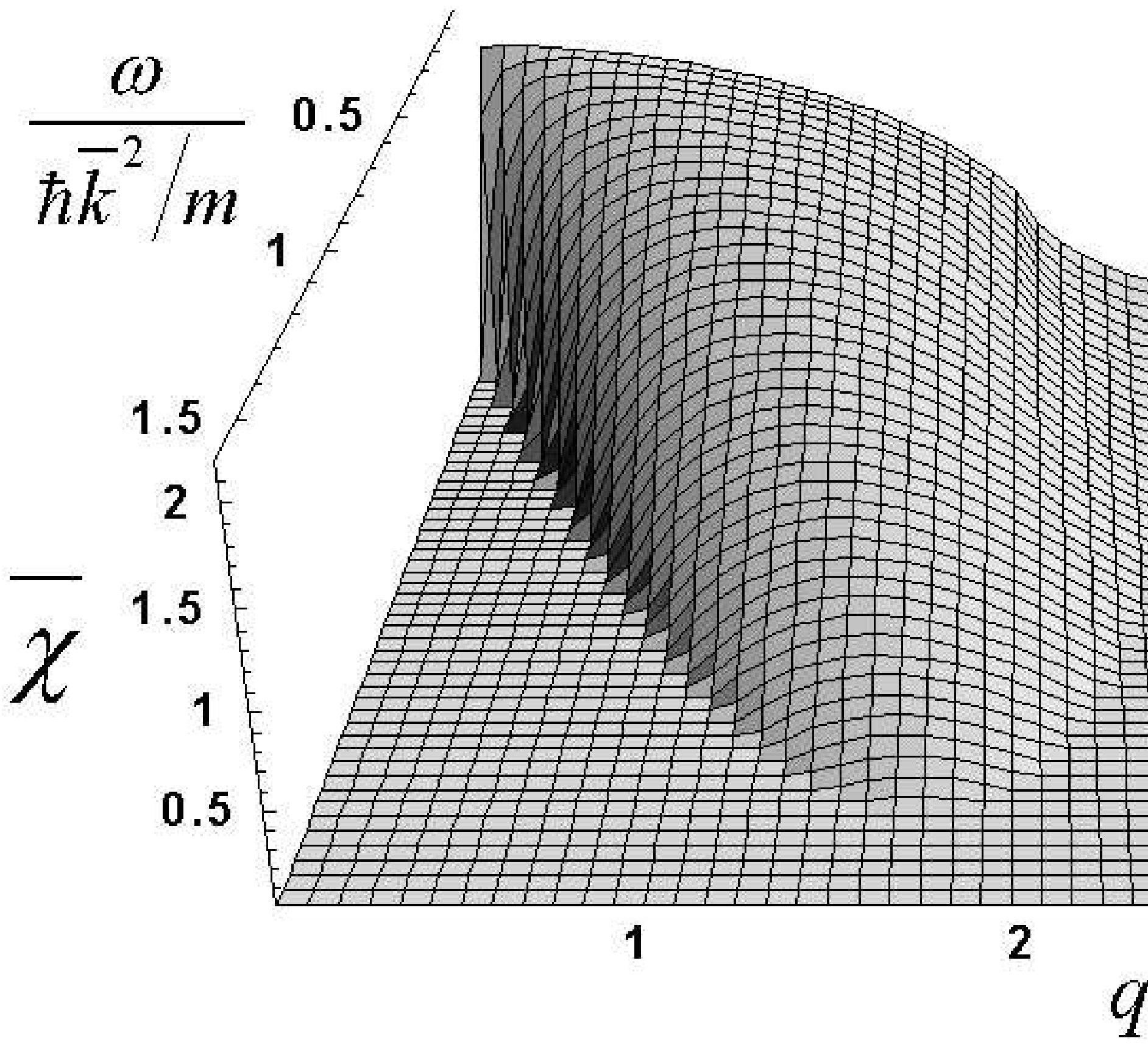

Fig. 7 (chi bar), K. Esfarjani et al. 


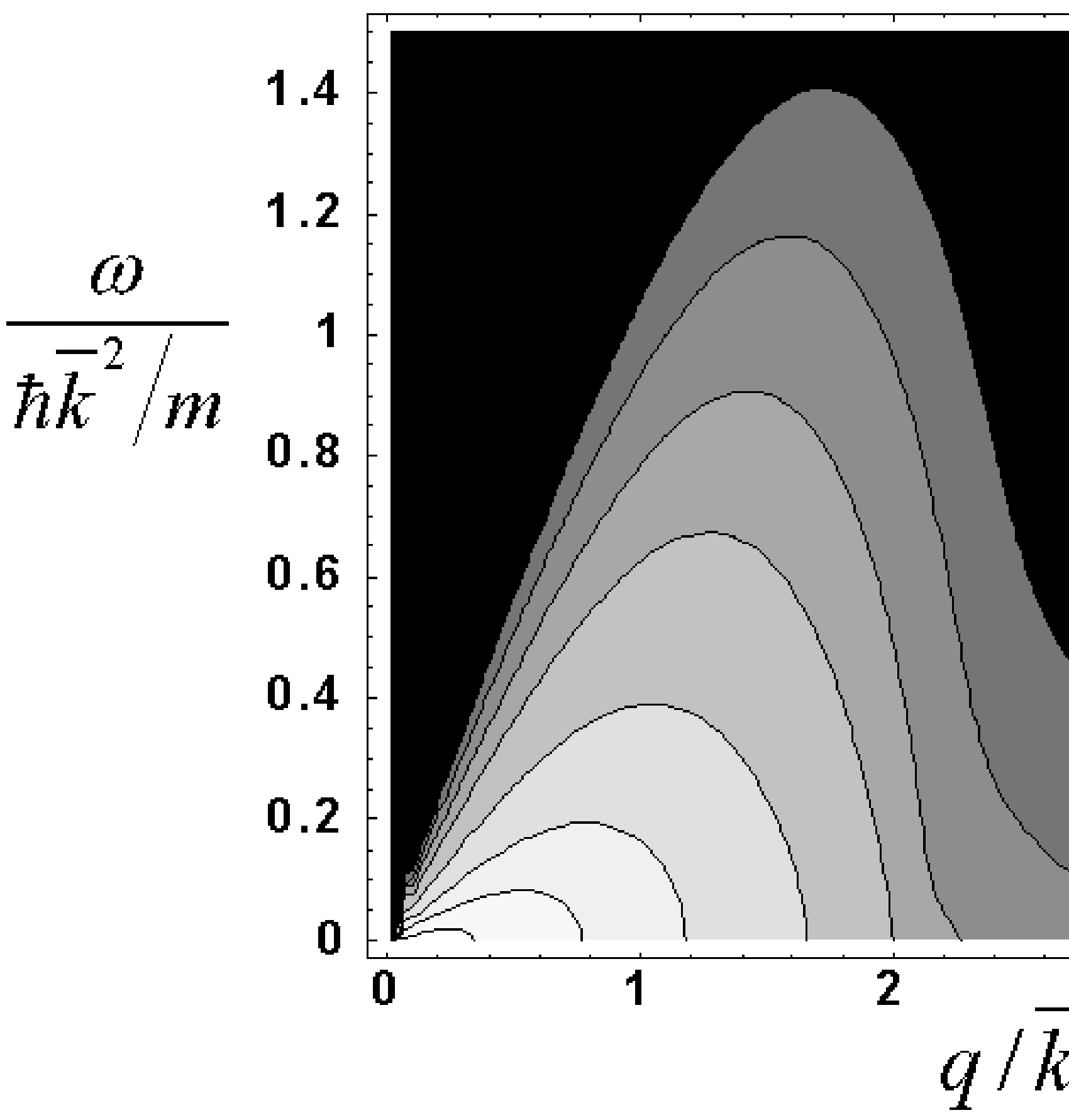

Fig. 8 (contours of chi bar), K. Esfarjani et al. 